\title{
Grafos associados a Quadrados Latinos e Grupos
}

\section{Graphs associated to Latin Squares and Groups}

Mateus Alegri

Universidade Federal de Sergipe (UFS)

Departamento de Matemática (DMAI), Itabaiana, SE, Brasil

allegri.mateus@gmail.com

Wagner Ferreira Santos

Universidade Federal de Sergipe (UFS)

Departamento de Matemática (DMAI), Itabaiana, SE, Brasil

wagnermaths@gmail.com

Samuel Brito Silva

Universidade Federal de Sergipe (UFS)

Departamento de Matemática (DMAI), Itabaiana, SE, Brasil

s.complexo@hotmail.com

\section{Informações do Artigo}

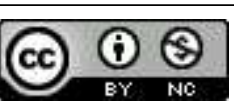

Histórico do Artigo

Submissão: 21 de fevereiro de 2018.

Aceite: 14 de maio de 2018.

\section{Palavras-chave}

Quadrados Latinos

Quasigrupos

Grafos

\section{Resumo}

Neste trabalho, abordaremos algumas conexões existentes entre as teorias dos grupos finitos e grafos. Na primeira seção, faremos uma breve introdução ao conceito de quadrados latinos e quasigrupos finitos, estrutura matemática que generaliza a estrutura de grupos. Na segunda seção, exibiremos um critério para que um quadrado latino seja a tabela de um grupo. Na última parte do trabalho, estudaremos resultados envolvendo grafos, sendo alguns destes obtidos pelos autores.
Keywords

Latin Squares

Quasigroups

Graphs

\section{Abstract}

In this work we will discuss some connections between finite group and graph theories. In the first section we will make a brief introduction to the concept of Latin squares and finite quasigroups, mathematical structure that generalizes the structure of groups. In the second section we will show a criterion for a Latin square to be the table of a group. In the last part of the work we will study results involving graphs, some of them obtained by the authors.

\section{Introdução}

Neste artigo, exploraremos conexões entre grupos, grafos e quadrados latinos, de modo que é requerido apenas ao leitor possuir conhecimentos básicos de grupos de permutação e grafos que 
podem ser encontrados nas referências Gonçalvez (2015) e Balakrishnan (2014). Nosso objetivo é encontrar condições em um quadrado latino para que este seja (ou não) a tabela de um grupo. Para isso, associamos um quadrado latino a um grafo via uma representação diferente da encontrada na literatura, que facilita a visualização de certas propriedades, como a obtenção de subgrafos que representam subgrupos.

Definição 1.1. Um quadrado latino de ordem $n$ é uma matriz $n$ por $n$ na qual um elemento não pode se repetir em linha nem em coluna.

Neste trabalho, consideramos as entradas de um quadrado latino $L$ de ordem $n$ em $\{1,2, \ldots, n\}$.

Exemplo 1.2. Os dois quadrados a seguir são exemplos de quadrados latinos de ordem 3 e 4 .

$\begin{array}{lllllll}1 & 3 & 2 & 1 & 2 & 3 & 4 \\ 2 & 1 & 3 & 2 & 1 & 4 & 3 \\ 3 & 2 & 1 & 3 & 4 & 1 & 2 \\ 4 & 3 & 2 & 1\end{array}$

Um quadrado latino reduzido $L$ de ordem $n$, é um quadrado latino em que a primeira linha e primeira coluna aparece na ordem natural: $1,2, \ldots, n$. O quadrado latino da direita é um exemplo de um quadrado latino reduzido.

A seguir, forneceremos a definição de uma estrutura algébrica que generaliza a noção de grupos e, de certa forma, a de quadrados latinos.

Definição 1.3. Dada * uma operação binária em um conjunto $Q$, o par $(Q, *)$ é um quasigrupo se para todo par $a, b \in Q$, as equações $a * x=b$ e $y * a=b$ são unicamente solúveis para $x$ e y em $Q$.

Devido às leis de cancelamento, todo grupo é um quasigrupo.

\section{Quasigrupos e Quadrados Latinos}

A proposição a seguir nos diz que se a operação do quasigrupo $Q$ é associativa para todos os elementos de $Q$, então $Q$ tem estrutura de grupo. A demonstração da Proposição 2.1 pode ser encontrada em Alegri e Silva (2017). 
Proposição 2.1. Seja $(Q, *)$ um quasigrupo. Se esta estrutura é associativa então $(Q, *)$ é um grupo.

Segue da proposição acima que toda tabela de Cayley de um grupo finito é um quadrado latino. Deste modo, obtemos o seguinte resultado:

Corolário 2.2. A tabela de Cayley de um grupo de ordem n é um quadrado latino de ordem $n$.

A recíproca deste corolário nem sempre é verdadeira. Exemplos de quadrados latinos que não são tabelas de Caley de grupos podem ser encontrados em Alegri e Silva (2017).

O próximo teorema fornecerá um critério simples que estabelece quando um quadrado latino representa, ou não, a tabela de um grupo. Este critério é creditado a Suschkewitch (1929) e discutido em Siu (1991).

Teorema 2.3. (Critério de Siu/Suschkewitch) Um quadrado latino é a tabela de um grupo se, e somente se, a composição de duas linhas é uma linha do quadrado.

Exemplo 2.4. Consideremos o quadrado latino $L$ dado por

$\begin{array}{llllll}1 & 2 & 3 & 4 & 5 & 6 \\ 2 & 1 & 4 & 5 & 6 & 3 \\ 3 & 6 & 1 & 2 & 4 & 5 \\ 4 & 5 & 6 & 1 & 3 & 2 \\ 5 & 3 & 2 & 6 & 1 & 4 \\ 6 & 4 & 5 & 3 & 2 & 1\end{array}$

Calculando a composição da última e quarta linhas, denotada por $l_{6} \circ l_{4}$, obtém-se:

$$
\left(\begin{array}{llllll}
1 & 2 & 3 & 4 & 5 & 6 \\
6 & 4 & 5 & 3 & 2 & 1
\end{array}\right) \circ\left(\begin{array}{llllll}
1 & 2 & 3 & 4 & 5 & 6 \\
4 & 5 & 6 & 1 & 3 & 2
\end{array}\right)=\left(\begin{array}{llllll}
1 & 2 & 3 & 4 & 5 & 6 \\
3 & 2 & 1 & 6 & 5 & 4
\end{array}\right)
$$

Onde a permutação resultante não é nenhuma linha do quadrado latino, logo, pelo critério de Siu/Suschkewitch, L não é a tabela de Cayley de um grupo.

\section{Grafos e Quadrados Latinos}

Nesta seção, faremos construções de quadrados latinos utilizando grafos, e a partir destas, obteremos propriedades envolvendo grupos, quasigrupos e quadrados latinos. Ressaltamos que as 
construções de grafos de arestas não coloridas associadas aos quasigrupos desta seção são originais, bem como os lemas 3.4 e 3.5, as proposições 3.7 (Teste da linha inversa) e 3.8 (A Permutação Total) e o teorema 3.11. Para as primeiras considerações, utilizaremos uma família de grafos bipartidos com arestas coloridas, muito úteis na busca de soluções de problemas de correspondência ${ }^{1}$. Nestes grafos, os vértices podem ser particionados em conjuntos disjuntos $U$ e $V$ onde nenhuma aresta liga dois vértices em $V$ ou em $U$. Um grafo deste tipo, no qual o número de elementos de $U$ denotado por $n$, é igual ao número de elementos de $V$, e todo vértice de $U$ é ligado por uma aresta a todo vértice de $V$ é chamado de um grafo bipartido completo de ordem $n$ e denotado por $K_{n, n}$. Para nosso estudo, as arestas do $K_{n, n}$ serão coloridas com $n$ cores de modo que cada vértice possua uma aresta de cada cor incidente. Lembramos que um 1-fator de um grafo $(V, E)$ é um subgrafo $\left(V, E^{\prime}\right)$, tal que cada vértice tem apenas uma aresta incidindo nele. Se $E$ pode ser particionado em subconjuntos disjuntos de tal maneira que $(V, E)$ se decompõe em 1-fatores, dizemos que 0 grafo $(V, E)$ é 1-fatorável. A partir da 1-fatoração de grafos, podemos relacionar grafos e quadrados latinos. A demonstração da proposição a seguir está disponível em Laywine e Mullen (1998).

Proposição 3.1. Um quadrado latino de ordem n é equivalente a uma 1-fatoração do grafo completo $K_{n, n}$.

Vale observar que a recíproca desta proposição é válida, ou seja, uma 1-fatoração do grafo completo $K_{n, n}$ é um quadrado latino.

Exemplo 3.2. Nas figuras 1 e 2, seguem a construção linha a linha do quadrado latino L e o grafo representando o próprio $L$. Aqui a cor preta representa o número 1, as cores vermelho, verde e roxo representam os números 2,3 e 4 , respectivamente.

\begin{tabular}{l|llll}
. & 1 & 2 & 3 & 4 \\
\hline 1 & 1 & 2 & 3 & 4 \\
2 & 2 & 1 & 4 & 3 \\
3 & 3 & 4 & 1 & 2 \\
4 & 4 & 3 & 2 & 1
\end{tabular}

\footnotetext{
${ }^{1}$ Um exemplo de um problema de correspondência é exibido a seguir. Suponha que haja $n$ candidatos e $n$ empregos distintos, onde cada candidato se inscrever para um emprego. Fazendo a correspondência entre a aresta que liga uma pessoa a um emprego, obtemos uma solução para este problema de correspondência, que é representado por um grafo bipartido.
} 
Figura 1 - Grafos representando linhas do quadrado $L$.

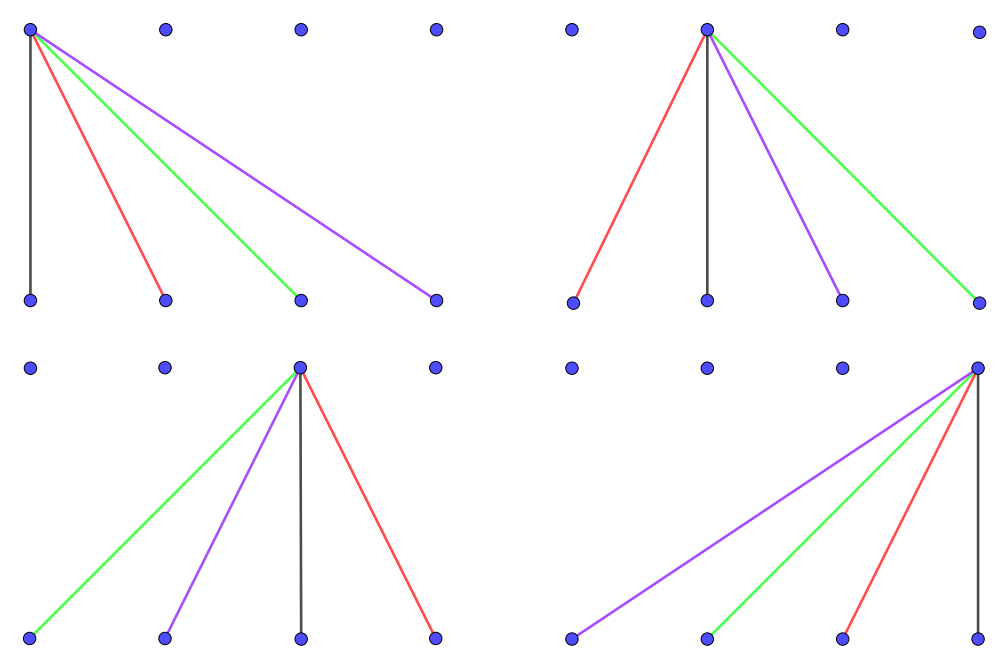

Fonte: Elaboração dos autores.

Figura 2 - Grafo de arestas coloridas representando $L$.

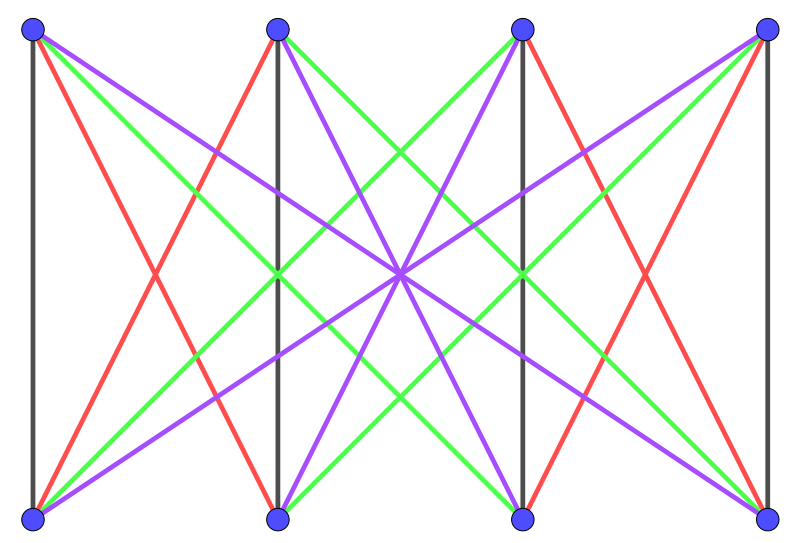

Fonte: Elaboração dos autores.

É claro que a representação de um quadrado latino via um grafo de arestas coloridas como feito no exemplo anterior é única. A partir da associação de um grafo e um quadrado latino como feita aqui vamos obter outra associação equivalente entre estes objetos, porém de maneira mais simples, não utilizando cores. Esta nova representação, criada pelos autores, será útil para obter condições necessárias para que um quasigrupo seja associativo e para obter subgrupos de um grupo finito.

Seja $L$ um quadrado latino de ordem $n$. Defina $h(L)$ como o grafo com $n(n+1)$ vértices dividido em $n+1$ colunas de $n$ elementos cada, e $n^{2}$ arestas. Arestas ligam um vértice da coluna $i$ a $i+1$, induzida pela $i$ ésima linha de $L$. Os vértices da coluna $i$ serão denotados por $v_{r, i}$. Este 
vértice será ligado ao $v_{s, i+1}$ se tivermos em $L$ que $i * r=s$. Denominamos o fator $f_{i}$ pelo subgrafo formado pelos vértices $v_{r, i}, v_{s, i+1}$, com $r, s \in\{1,2, \ldots, n\}$. Observe que sendo $L$ um quadrado latino, há somente duas arestas atingindo todo vértice do grafo $h$, e ainda esta representação é única e será chamada neste texto de representação gráfica 2.

Exemplo 3.3. Na esquerda da Figura 3, representamos o quadrado latino L gerado a partir da tabela de Cayley do grupo de Klein $V$ e, no centro, sua representação gráfica utilizando cores, que chamaremos de representação gráfica 1. A direita, temos a representação gráfica $h(L)$, que chamaremos de representação gráfica 2, da tabela do grupo de Klein.

Figura 3 - Representações gráficas do grupo de Klein.
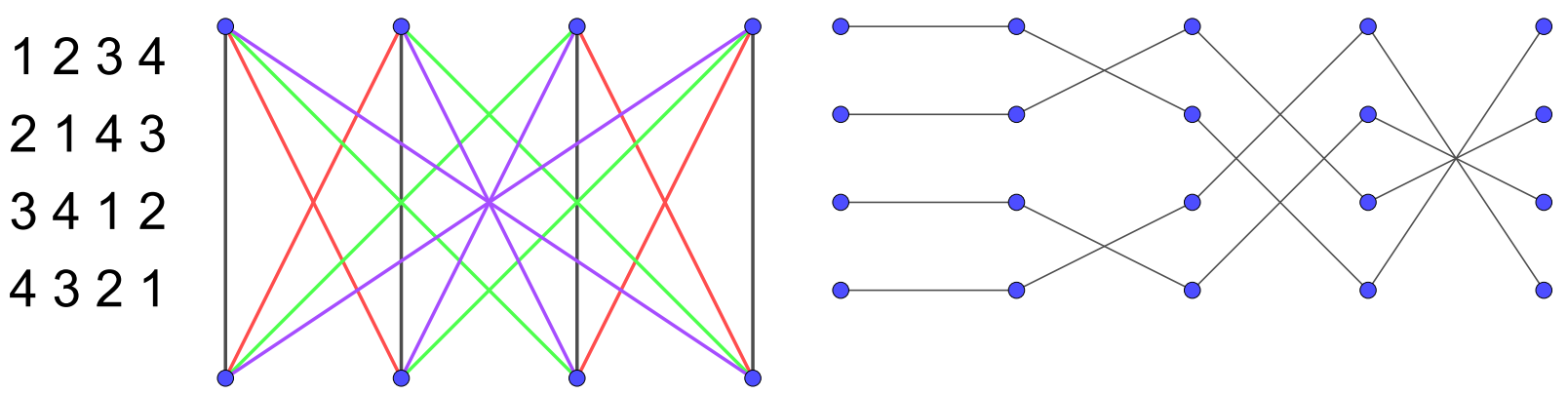

Fonte: Elaboração dos autores.

Dado $n$ um número natural, considere o conjunto $H_{n}$ de grafos como descritos na representação gráfica 2 de um quadrado latino. É relativamente fácil de ver que, devido à unicidade da representação abordada, cada grafo de $H_{n}$ representa um quasigrupo de mesma ordem, tornando verdadeiro o seguinte lema.

Lema 3.4. O número de grafos elementos do conjunto $H_{n}$ é igual ao número de quasigrupos de ordem n, contando os possíveis isomorfismos.

Por questões de simplicidade, vamos estabelecer duas condições iniciais que serão utilizadas neste trabalho.

1. A primeira linha $l_{1}$ de $L$ está em ordem natural, i.e., o quadrado latino $L$ é reduzido.

2. Todo elemento $i$ de um quasigrupo $Q$ possui inversa, i.e., em uma coluna $j$ e na linha $i$ de $L$, o elemento 1 aparece, bem como na linha $j$ e coluna $i$. 
Para o item 1, estamos cobrando que o quasigrupo possua elemento neutro (e assim $Q$ é um loop). A linha $l_{1}$ é chamada de linha neutra, porque a composição de $l_{1}$ com qualquer linha $l_{j}$ resulta na linha $l_{j}$. A fim de verificar o item 2., devemos apenas observar a posição dos $1^{\prime} s$ no quadrado $L$ de modo a garantir que o inverso à direita seja igual ao inverso à esquerda do elemento da primeira coluna de $L$.

No próximo lema, vamos estabelecer que dado um grupo $Q$ de ordem $n$, o conjunto de linhas de sua tabela de Cayley $l=\left\{l_{1}, l_{2}, \ldots, l_{n}\right\}$ munido com a operação de composição de linhas também tem estrutura de grupo. Obviamente, nestas condições, $Q$ é isomorfo a $l$, e a operação o em $l$ é dada por $l_{i} \circ l_{j}=l_{i * j}$. O propósito em fornecer o seguinte lema é estudar condições nos fatores de um grafo $h(L) \in H_{n}$, a fim de que $L$ seja uma tabela de Cayley de um grupo.

Lema 3.5. Tome $l=\left\{l_{1}, l_{2}, \ldots, l_{n}\right\}$ o conjunto de todas as linhas de um quadrado latino $L$, tabela de multiplicação do quasigrupo $Q$, respeitando as condições iniciais. O conjunto $l$ munido da operação de composição de linhas é um grupo, se e somente se, $Q$ é um grupo.

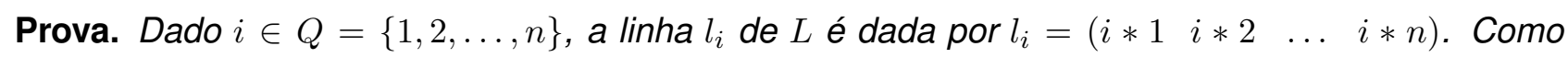
$L$ satisfaz as condições iniciais, o elemento 1 é o elemento neutro de $Q$, e a composição $l_{i} \circ l_{j}$ é dada por $m=(i *(j * 1) \quad i *(j * 2) \quad \ldots \quad i *(j * n))$. Desde que $m$ é uma linha de $L$ é $m=l_{i * j}=$ $((i * j) * 1 \quad(i * j) * 2 \quad \ldots \quad(i * j) * n) \in l$, e l é um grupo, então $Q$ é associativo, logo $Q$ é grupo.

Reciprocamente, se $Q$ é um grupo, então a composição de linhas é uma operação binária para os elementos de $l$, sendo $l_{1}$ o elemento neutro de $l$. Afirmamos que todos os elementos de $l$ possuem inversa em $l$. Com efeito, pela condição inicial 2., dada uma linha $l_{i}$ de $L$, a sua inversa é a linha $l_{i^{-1}}$, onde $i^{-1}$ é o inverso do elemento $i \in Q$. De fato, sendo $l_{i}=\left(\begin{array}{lll}i & i * 2 & i * 3 \quad \ldots \quad i * n\end{array}\right) e$ $l_{i^{-1}}=\left(\begin{array}{lllll}i^{-1} & i^{-1} * 2 & i^{-1} * 3 & \ldots & i^{-1} * n\end{array}\right)$, em uma coluna $k$ de $L$, temos pela lei da associatividade em $Q$ o seguinte fato: $l_{i} \circ l_{i^{-1}}(k)=i *\left(i^{-1} * k\right)=k$, e então $l_{i} \circ l_{i^{-1}}=l_{1}$.

Dada uma linha $l_{i}$ em um quadrado latino $L$, sabemos que esta linha é representada pelo i-ésimo fator do grafo $h \in H_{n}$. A operação de composição de duas linhas $l_{i}$ e $l_{j}$, pode ser representada graficamente pelos caminhos resultantes que partem dos vértices de $l_{i}$ e alcançam os vértices adjacentes de $l_{j}$. Na Figura 4, temos a representação da operação de composição de fatores, que, no caso, representam a operação de composição das linhas $l_{1}=\left(\begin{array}{llll}1 & 3 & 2 & 4\end{array}\right)$ e $l_{2}=\left(\begin{array}{llll}2 & 4 & 3 & 1\end{array}\right)$ gerando a linha (2 $\left.3 \begin{array}{lll}2 & 4 & 1\end{array}\right)$. 
Figura $4-A$ composição dos fatores à esquerda gera o fator à direita.

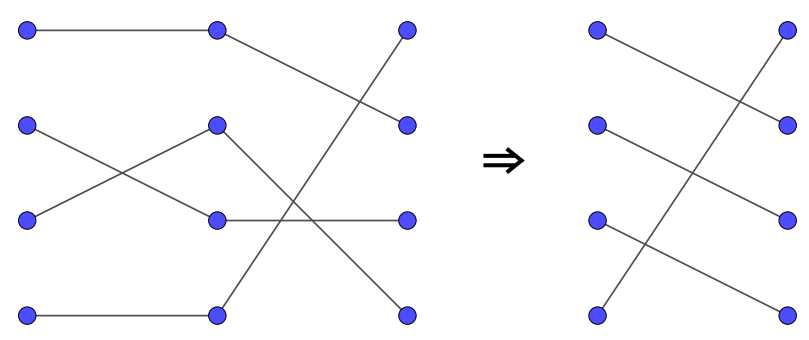

Fonte: Elaboração dos autores.

Dizer que este i-esimo fator é inversível é o mesmo que dizer que a linha $l_{i}$ é inversível em $(l, \circ)$. Graficamente, um fator $k$ é inversível se existe um fator $j$ em $h$ tal que os caminhos saindo de cada vértice $i$ no fator $k$ atinge o vértice $i$ no fator $j$.

Exemplo 3.6. Na Figura 5, temos a representação gráfica 2 da tabela $L$ do grupo $\mathbb{Z}_{5}$. Denotamos por $l_{1}=\left(\begin{array}{lllll}1 & 2 & 3 & 4 & 5\end{array}\right), l_{2}=\left(\begin{array}{lllll}2 & 3 & 4 & 5 & 1\end{array}\right), l_{3}=\left(\begin{array}{lllll}3 & 4 & 5 & 1 & 2\end{array}\right), l_{4}=\left(\begin{array}{llllll}4 & 5 & 1 & 2 & 3\end{array}\right)$ e $l_{5}=\left(\begin{array}{lllll}5 & 1 & 2 & 3 & 4\end{array}\right)$.

Figura 5 - Representação gráfica da tabela do $\mathbb{Z}_{5}$.

12345

23451

34512

45123

51234

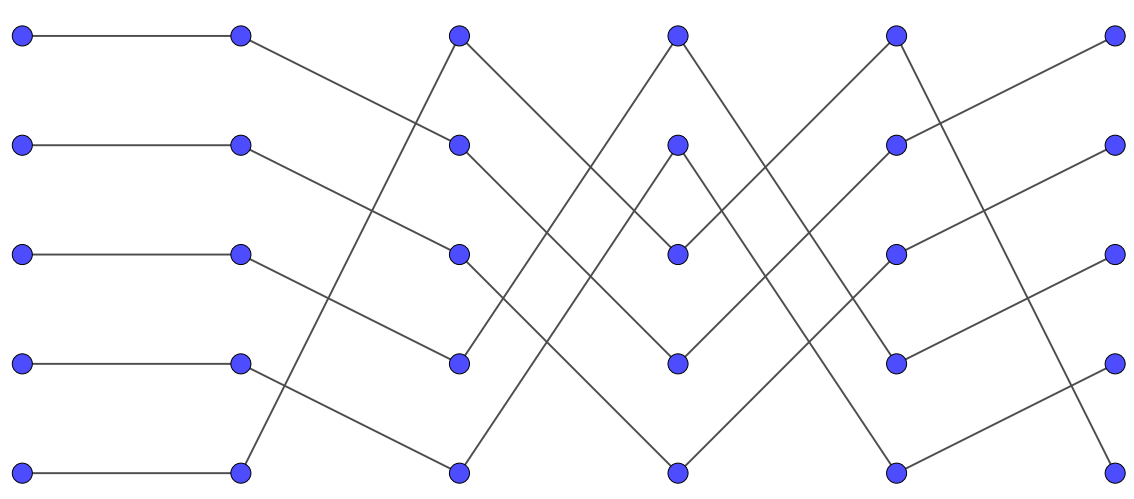

Fonte: Elaboração dos autores.

Note que $l_{1}^{-1}=l_{1}, l_{2}^{-1}=l_{5}$ e $l_{3}^{-1}=l_{4}$. Na Figura 6 , segue a representação gráfica da operação de composição das linhas 2 e 5 do quadrado, via caminhos nos respectivos grafos. 
Figura 6 - Representação gráfica da composição de linhas.

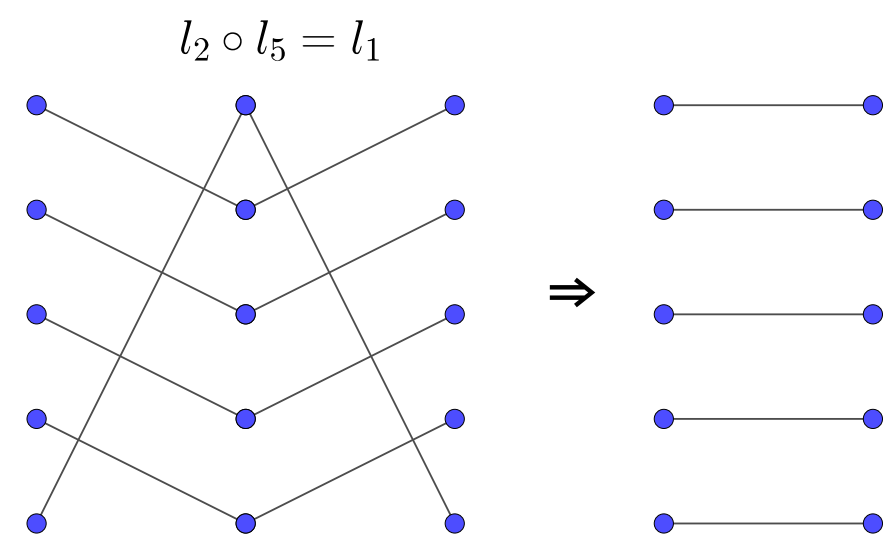

Fonte: Elaboração dos autores.

Pelo Lema 3.5, se um quadrado $L$ satisfaz as condições iniciais 1 e 2 e uma dada linha não possui linha inversa, a associatividade não está assegurada para os elementos de $Q$. De fato, dado uma linha $l_{i}=\left(\begin{array}{lllll}i * 1 & i * 2 & \ldots & i * n\end{array}\right)$, como existe a linha $l_{i^{-1}}=\left(\begin{array}{llll}i^{-1} * 1 & i^{-1} * 2 \quad \ldots \quad i^{-1} * n\end{array}\right)$, existe um $k \in Q$ tal que $i *\left(i^{-1} * k\right) \neq\left(i * i^{-1}\right) * k$. Em seguida, formalizamos o chamado Teste da Linha Inversa que requer no máximo $n$ operações de composições a serem realizadas.

Proposição 3.7. (Teste da linha inversa)

Tome $h(L) \in H_{n}$ representando a tabela $L$ do quasigrupo $Q$, satisfazendo as condições iniciais 1 e 2. Se para um fator em $h$ não existe o fator inverso, então $Q$ não é associativo.

Neste segundo teste, o teste da Permutação Total, apresentamos uma condição necessária, utilizando $n$ composições de linhas, para verificar a associatividade de $Q$.

Proposição 3.8. (A Permutação Total) Tome $h$ um grafo de $H_{n}$ representando a tabela de multiplicação $L$ de um quasigrupo $Q$, não necessariamente respeitando as condições iniciais 1 e 2. Partindo do vértice $i$ no primeiro fator de $h$, considere um caminho no grafo até o último vértice no último fator de $h$. Fazendo isto para todos os vértices do primeiro fator, se os caminhos correspondentes não geram um fator do grafo $h$, então $Q$ é não associativo.

Prova. Considerando os caminhos no grafo $h$, como descritos no enunciado da proposição, a operação nos fatores de $h$ é equivalente a se calcular $n$ composições nas linhas de $L$, como $\left(\ldots\left(\left(l_{1} \circ l_{2}\right) \circ l_{3}\right) \ldots\right) \circ l_{n}$, nesta ordem. De fato, se o correspondente fator neste processo, cha- 
mado de Permutação Total, não forma um fator de $h$, então a composição de linhas não é fechada em $L$ implicando no fato de que $Q$ não é associativo.

Exemplo 3.9. Considere a tabela de um quasigrupo $Q$ como mostrada na Figura 7. As arestas partindo dos números de 1 a 5 são coloridos com as respectivas cores: preto, verde, vermelho, ciano e amarelo, para facilitar a visualização. A permutação total fornece o grafo representado na Figura 7 , que não é um fator do grafo da Figura 8. A permutação total representada na Figura 7 é equivalente à permutação ( $\left.\begin{array}{lllll}3 & 4 & 5 & 2 & 1\end{array}\right)$ que não é linha do quadrado L.

Figura 7 - A Tabela de $L$ à esquerda e o grafo correspondente, à direita.

12345

25413

31254

43521

54132

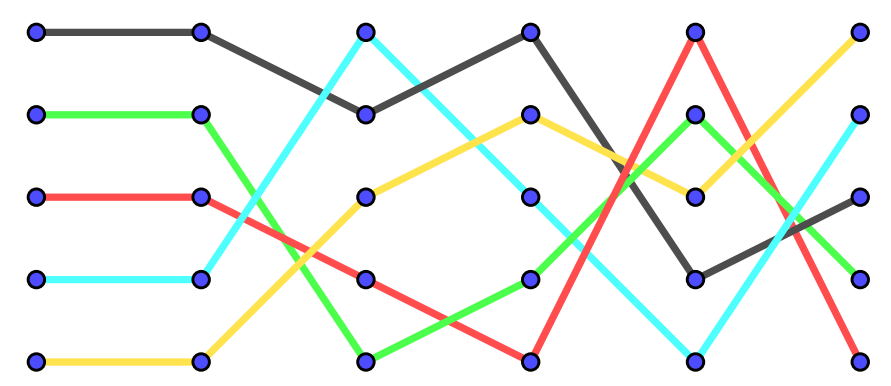

Fonte: Elaboração dos autores.

Figura 8 - Grafo resultante da permutação total em $L$.

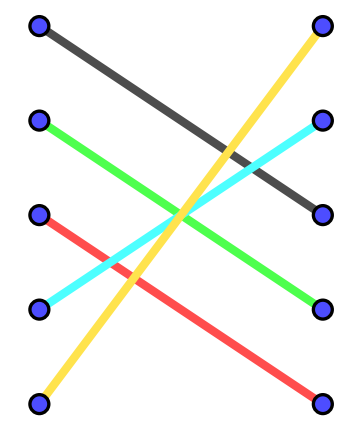

Fonte: Elaboração dos autores.

Observação 3.10. As condições discutidas nesta seção são apenas suficientes. Na Figura 9 é fornecido um exemplo de uma tabela $L$ que não é tabela de Cayley de um grupo em que são satisfeitos os critérios da linha inversa e da permutação total. Note que o quasigrupo $Q$ associado a esta tabela não é associativo pois $(2 * 3) * 4=3 \neq 2 *(3 * 4)=2$. 
Figura 9 - Representação gráfica 2 de uma tabela de um quasigrupo.

123456

215634

362145

431562

546321

654213

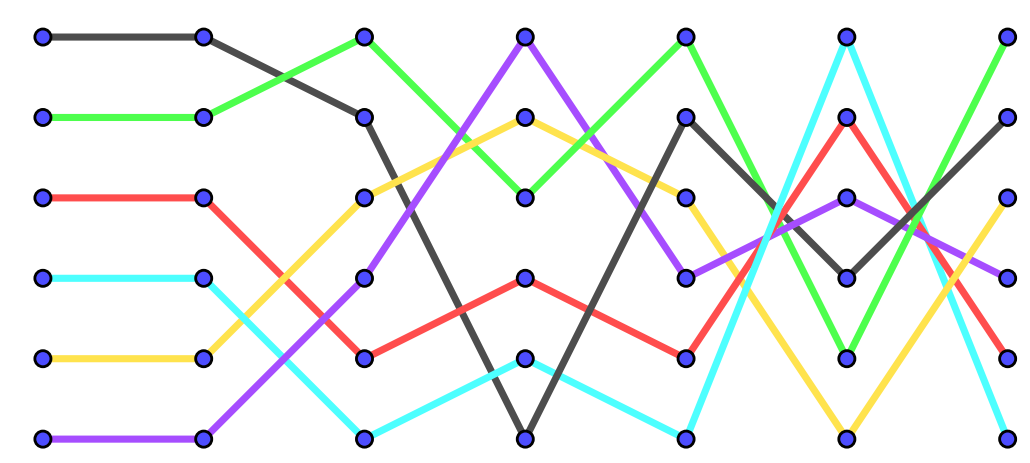

Fonte: Elaboração dos autores.

Baseado no critério de Siu/Suschkewitch (Teorema 2.3), podemos encontrar todos os grafos em $H_{n}$ que representam tabelas de Cayley de grupos de ordem $n$.

Teorema 3.11. Seja $Q$ um quasigrupo de ordem n com tabela de multiplicação $L$ satisfazendo a condição inicial 1, sendo representado pelo grafo $h(L) \in H_{n}$. Se a composição de dois fatores quaisquer em $h(L)$ ainda é um fator de $h(L)$, então $Q$ é um grupo.

Exemplo 3.12. Na Figura 10, tem-se a representação do grafo $h$ do grupo $S_{3}$, cuja tabela de Cayley é exibida na esquerda desta. Note que o correspondente grafo $h$ de $S_{3}$ é fechado em relação à composição de fatores. Na Figura 11, segue o esquema da composição dos fatores quatro e seis de $S_{3}$, que representam as linhas 4 e 6 da tabela de Cayley do grupo, gerando o terceiro fator.

Figura 10 - Grafo de $H_{6}$ representando a tabela do $S_{3}$.

123456

231564

312645

465132

546213

654321

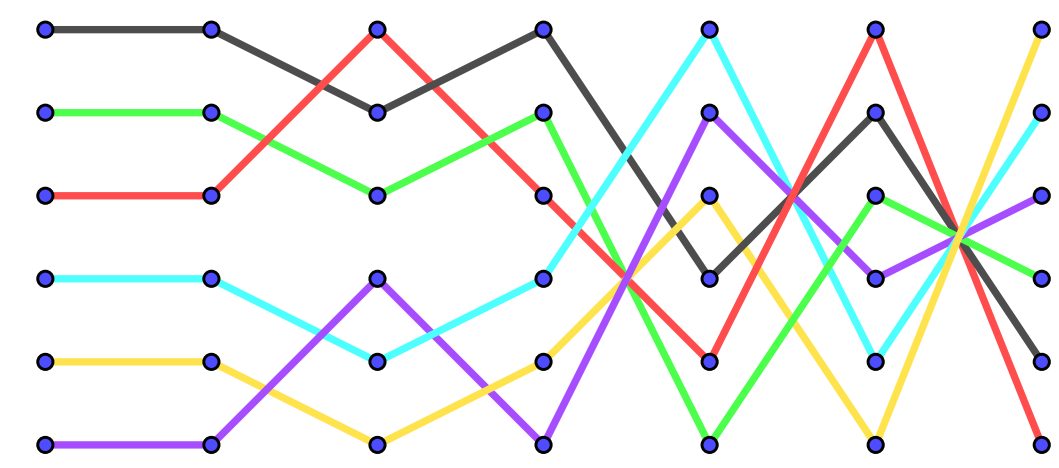

Fonte: Elaboração dos autores. 
Figura $11-$ Grafo representando a operação $l_{4} \circ l_{6}$.

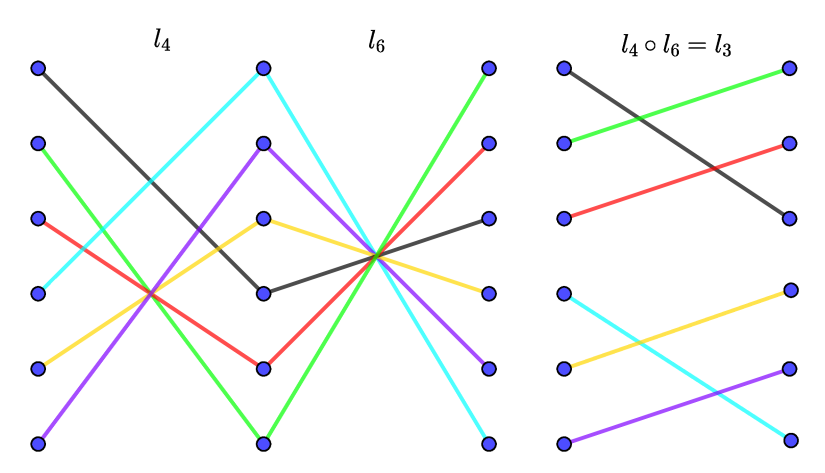

Fonte: Elaboração dos autores.

\section{Subgrupos e Grafos}

Nesta seção, encontraremos condições gráficas para que dado um subgrafo $h^{\prime}$ de $h \in H_{n}$, satisfazendo a condição do teorema 3.11, seja associado um conjunto $Q^{\prime} \subset Q$, de modo que $Q^{\prime}$ tenha estrutura de grupo. Todos os resultados desta seção são originais.

Teorema 4.1. Seja $G$ um grupo de ordem $n$ com tabela $L$ associado ao grafo $h \in H_{n}$. O subconjunto $G^{\prime}$ com $n^{\prime}$ elementos em que $1 \in G^{\prime}$ é um subgrupo de $G$ se, e somente se, o grafo $h^{\prime}$ associado a $G^{\prime}$ é um subgrafo de $h$ que há exatamente $n^{\prime}$ fatores com vértices em $G^{\prime}$ e $n^{\prime}\left(n^{\prime}-1\right)$ arestas, onde todo vértice $i$ é conectado em algum fator ao vértice 1.

Prova. Seja $h^{\prime}$ um subgrafo de $h \in H_{n}$ satisfazendo as condições expostas no enunciado do teorema. Como $h^{\prime}$ é subgrafo, afirmamos que $h^{\prime}$ representa um quadrado latino $L^{\prime}$ que é a tabela de Cayley do grupo $G^{\prime}$. De fato, devido à definição do subgrafo $h^{\prime}, G^{\prime}$ é fechado em relação à operação herdada de $G$ e como todo vértice de $h^{\prime}$ é conectado ao vértice $i$, então todo elemento de $G^{\prime}$ possui inversa em $G^{\prime}$, provando que $G^{\prime}$ é um subgrupo de $G$.

Reciprocamente, dado $G$ um grupo e $G^{\prime}$ um subgrupo de $G$, existem grafos $h$ e $h^{\prime}$ de $G$ e $G^{\prime}$, respectivamente. Desde que os elementos de $G$ são elementos de $G$, segue que os vértices de $h^{\prime}$ são vértices de $h$. Além disso, como $G^{\prime}$ é um subgrupo de $G$, as arestas de $h^{\prime}$ são arestas de $h$, e portanto, $h^{\prime}$ é subgrafo de $h$. 
Exemplo 4.2. Seguem nas figuras 12 e 13 os grafos $h$ e $h^{\prime}$, que representam o grupo $G \cong Q_{8}$, onde $Q_{8}$ denota o grupo dos quatérnions seu subgrupo $G^{\prime}=\{1,2,3,4\} \cong \mathbb{Z}_{4}$, respectivamente.

Figura 12 - Grafo de $H_{8}$ representando a tabela de $Q_{8}$.

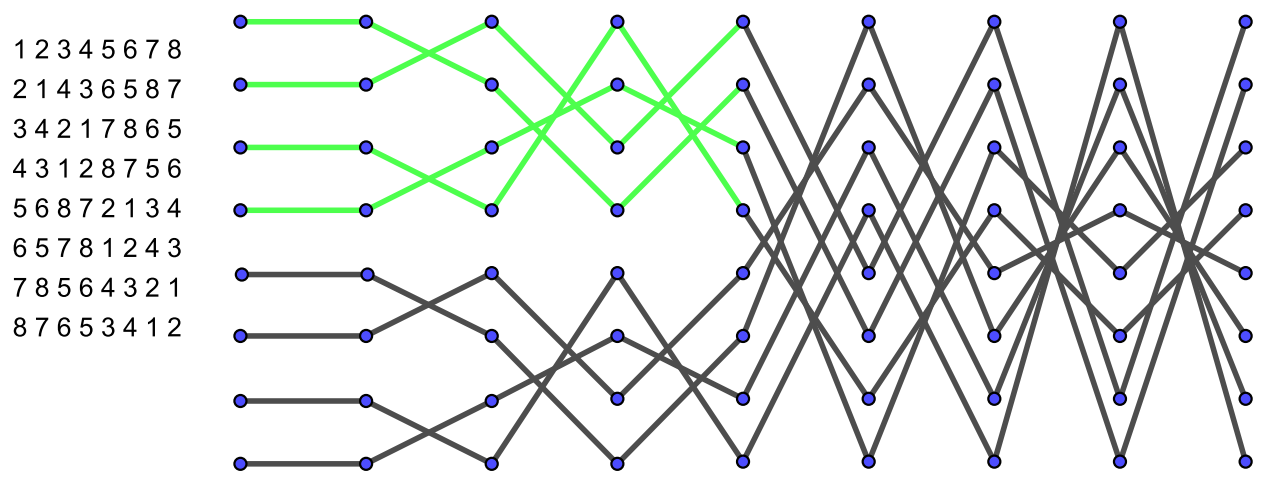

Fonte: Elaboração dos autores.

Figura 13 - Grafo representando a tabela de $G^{\prime} \subset Q_{8}$.

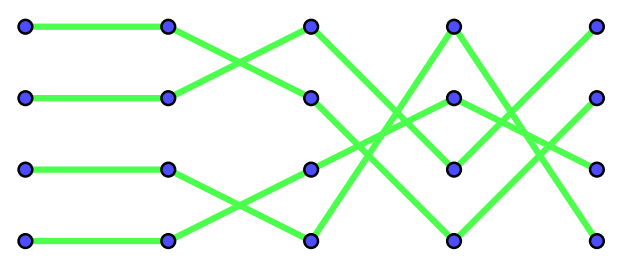

Fonte: Elaboração dos autores.

Definimos a representação gráfica 3 a partir da coloração das arestas da representação gráfica 2 induzida pela representação gráfica 1. Com efeito, a aresta ligando o vértice $i$ ao vértice $j$ terá a cor $k$ desde que o elemento $k$ apareça na linha $i$ e coluna $j$ do quadrado $L$. Denotamos o conjunto destes grafos coloridos vinculados a quasigrupos de ordem $n$ por $C_{n}$. Enquanto que denotamos um elemento de $C_{n}$ ao quasigrupo $Q$ por $c(Q) \in C_{n}$.

Segue a representação gráfica 3 do grupo de Klein induzida pelas representações gráficas da Figura 3. 
Figura 14 - Representação gráfica do grupo de Klein.

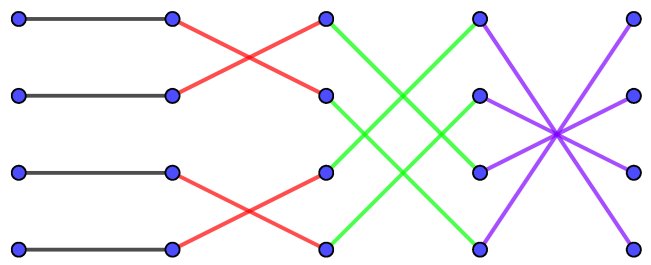

Fonte: Elaboração dos autores.

Teorema 4.3. Seja $G$ um grupo de ordem n cuja tabela de Cayley é L e sua representação gráfica 3 é $c(G)$. O conjunto $G^{\prime}=\left\{i_{1}, i_{2}, \ldots, i_{m}\right\} \subset G$, onde $i_{1}=1$, é um subgrupo de $G$ se em todos os fatores $k \in\left\{i_{1}, i_{2}, \ldots, i_{m}\right\}$ de $c(G)$, as arestas de $c\left(G^{\prime}\right)$ tem cores associadas aos elementos de $G^{\prime}$.

Prova. Dado um subconjunto $G^{\prime}$ de $G$, este sendo associado ao grafo colorido $c\left(G^{\prime}\right)$, dizer que as arestas de $c\left(G^{\prime}\right)$ são ligados entre si possuem cores em associados aos elementos de $G^{\prime}$ significa que a operação herdada por $G$ é fechada para os elementos de $G^{\prime}$. Como $1 \in G^{\prime}$ e sendo $G$ um grupo e todo elemento $i \in G^{\prime}$ é ligado a 1 no fator $i$, então todo elemento $i \in G^{\prime}$ possui inversa em $G^{\prime}$, caracterizando que $G^{\prime}$ possui estrutura de grupo.

Exemplo 4.4. Como vemos na Figura a seguir, temos uma representação gráfica da tabela do grupo $Q_{8}$ utilizando sete cores e uma linha pontilhada para as arestas. O conjunto $\{1,2,3,4\}$ forma um subgrupo de $Q_{8}$ isomorfo a $\mathbb{Z}_{4}$, e é representado pelo subgrafo da Figura 16.

Figura 15 - Cor 1: preto, 2: vermelho, 3: verde, 4: roxo, 5: amarelo, 6: preto tracejado, 7: Iaranja e 8: cinza.
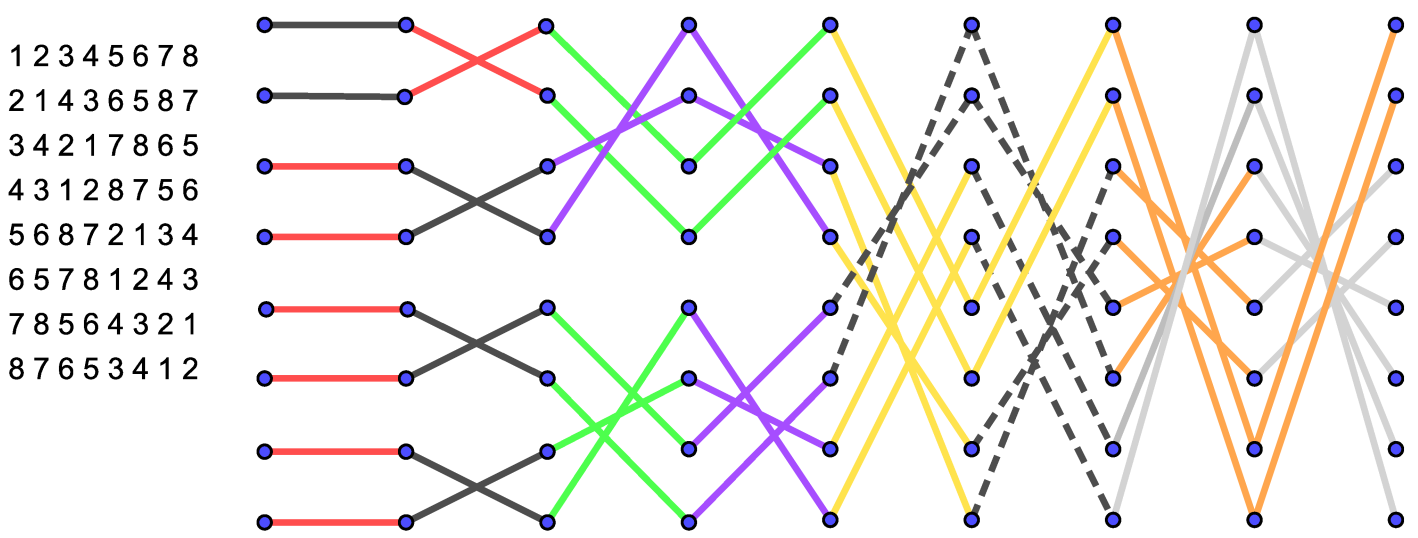

Fonte: Elaboração dos autores. 
Figura 16 - Cor 1: preto, 2: vermelho, 3:verde, 4:roxo.

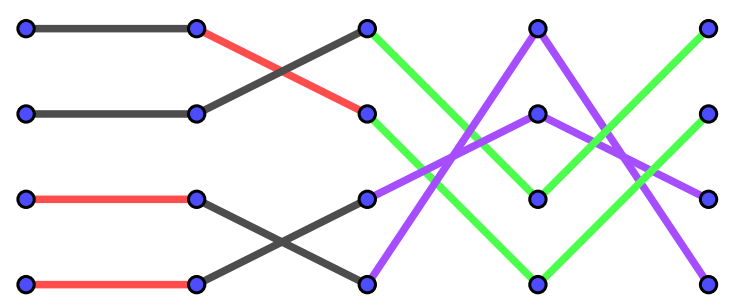

Fonte: Elaboração dos autores.

Os outros subgrupos próprios de $Q_{8}$ são $\{1,2\},\{1,2,3,4\},\{1,2,5,6\}$ e $\{1,2,7,8\}$ que podem ser observados facilmente na Figura acima.

\section{Considerações Finais}

Neste artigo, foram propostos seis novos resultados relacionando elementos de matemática discreta nos tópicos de teoria dos grupos e grafos. Destacamos a importância das duas condições necessárias descritas nas proposições 3.7 e 3.8 devido ao fato de haver muito mais quadrados latinos do que estruturas de grupos associados a estes. Como, por exemplo, existem apenas 5 grupos de ordem $n=8$ e aproximadamente $2,51 \times 10^{56}$ quadrados latinos desta mesma ordem. Deste modo, estas condições servem como testes efetivos em muitos casos.

Esperamos que este trabalho seja útil para propor estudos mais avançados sobre as relações entre grupos e quasigrupos finitos.

\section{Referências}

ALEGRI, M.; SILVA, S. B. Sobre Sudokus e Grupos. Revista Sergipana de Matemática e Educação Matemática, v. 2, p. 51-63, 2017.

BALAKRISHNAN, V. K. Introductory Discrete Mathematics. New York: Dover Books, 2014.

GONÇALVEZ, A. Introdução à Álgebra 5. ed. Rio de Janeiro: IMPA, 2015.

LAYWINE, C. F.; MULLEN, G. L. Discrete Mathematics Using Latin Squares. New York: Wiley Interscience Publication, 1998. 
SIU, M-K. Which Latin Squares are Cayley Tables? The American Mathematical Monthly, v. 98, n. 7, p. $625-627,1991$.

SUSCHKEWITCH, A. On a generalization of the associative law. Transactions American Mathematical Society, v. 31, p. 204-214, 1929. 